\title{
What Effect Does an Animal Robot Called CuDDler Have on the Engagement and Emotional Response of Older People with Dementia? A Pilot Feasibility Study
}

\author{
Wendy Moyle ${ }^{1,2,4}$ (D) Cindy Jones ${ }^{1,4} \cdot$ Billy Sung $^{1,4}$ - Marguerite Bramble ${ }^{1,4}$. \\ Siobhan O'Dwyer ${ }^{1,2,4}$ • Michael Blumenstein ${ }^{3}$ • Vladimir Estivill-Castro ${ }^{3}$
}

Accepted: 14 October 2015

(C) Springer Science+Business Media Dordrecht 2015

\begin{abstract}
The development of companion animal robots is of growing interest. These robots have recently been marketed to older adults with dementia as a means of encouraging social engagement and reducing behavioural and psychological symptoms of dementia. This paper outlines the results of a pilot study that sought to assess the feasibility and effect of using a robotic companion animal called CuDDler on engagement and emotional states of five older adults with dementia living in nursing home care. CuDDler is a prototype robot developed in Singapore. Despite their cognitive decline, the study participants raised a number of concerns regarding the feasibility and tolerability of CuDDler. The effectiveness of CuDDler was also limited in these participants, although one participant with visual agnosia benefited greatly from the one-on-one experience. The findings demonstrate the importance of companion robots being developed that are of an appropriate size, weight and shape for older people, including those with dementia, and a realistic animal shape that does not encourage thoughts of it being a toy. Our conclusions indicate the need for further studies on the development and use of companion robots, and investigation of the comparative benefits of social robots both compared to and in association with human interactions.
\end{abstract}

Wendy Moyle

w.moyle@griffith.edu.au

1 Menzies Health Institute QLD, Griffith University, 170 Kessels Road, Brisbane, Australia

2 Dementia Collaborative Research Centre - Carers and Consumers, QUT, Brisbane, Australia

3 Institute for Integrated and Intelligent Systems, Griffith University, 170 Kessels Road, Brisbane, Australia

4 Centre for Health Practice Innovation, Griffith University, Brisbane, Australia
Keywords Social robots - Dementia Older people . Long-term care $\cdot$ Companion animal

\section{Introduction}

\subsection{Dementia}

Dementia is a progressive syndrome whereby cognitive disability is secondary to some form of acquired brain damage - usually degenerative and occurring in older age, but not necessarily so. The most common form of dementia is Alzheimer's disease [1]. The prevalence of dementia is growing rapidly with a conservative estimate of 35.6 million people globally [1] and over 300,000 Australians [2] living with dementia. Dementia is one of the key reasons for entry into long-term care (LTC) and in Australia over $50 \%$ of this population has a diagnosis of dementia [2].

Deterioration in intellectual performance is accompanied by a significant decline in personal and social functions. Dementia affects an individual's ability to effectively communicate and to interact, as well as their memory, social judgement, mood and affect [3]. People with dementia can also exhibit behavioural and psychological deficits or excesses that cause stress for the person as well as those providing care. These include depression, visual perceptual deficits, psychosis, agitation, aggression, wandering, social and sexual dis-inhibition, as well as verbal outbursts, delusions, hallucinations, and anxiety [3,4]. Agitation is one of the most common behavioural disturbances, especially in Alzheimer's disease. Most definitions of agitation refer to aggressive, destructive, and resistive behaviours [3]. In addition, repeatedly asking questions, pacing, and repetitive motor behaviours are also acknowledged as being agitation. 
The most common agitated behaviour is verbal aggression [3].

A display of agitation can challenge the process of caring for people with dementia, can result in older people with dementia feeling lonely and socially isolated, and can be a result of unmet needs [5]. Behavioural and psychological symptoms of dementia such as agitation have traditionally been managed with pharmacological treatment such as antipsychotic medication [4]. The side effects of antipsychotic medication include sedation and an increase in falls, stroke and mortality [6,7]. These side effects have encouraged health professionals to trial psychosocial interventions to manage agitation [8]. Psychosocial interventions focus on addressing a person's unmet needs. Such needs include limited stimulation of the senses or a lack of meaningful conversation.

Residents in LTC often spend the majority of their time alone, and not engaged in meaningful activities [5,9]. They may also spend the best part of their days sitting waiting for something to happen [10]. A lack of stimulation can be particularly detrimental to people with dementia as it adversely affects their mood, increases their level of agitation and results in a high use of pharmaceutical interventions [6]. To counter these issues, researchers have been investigating the use of companion robotic animals as a means to comfort, engage, and stimulate social interaction with dementia [11-13].

\subsection{Companion Robotic Animals}

Companion robotic animals are designed to look, move and sound like an animal and they may be used to interact with people in a socio-emotional way. Examples of companion robots include Paro, a robotic seal [13] and Babyloid robot, a robotic baby [14]. Companion robots can provide an opportunity for an older person with dementia to be socially stimulated through interaction with the robot and/or the opportunity for the robot to be a tool for social interaction with other people in the environment [15].

In contrast to real animals, some advantages of robotic animals include: reduced burden on staff associated with the need to provide food or hygiene to a live animal; reduced risk for residents of being knocked over or bitten by a live animal; and reduced risk for a live animal of being overfed or unduly stressed by enthusiastic residents [12]. While we do not advocate the use of robots as a substitute for human company, robotic pets can fill those inevitable gaps during the day when the resident is alone and carers are otherwise occupied.

Companion robots may also enhance quality of life in older people with dementia [12]. Studies have reported improvements in relationships and loneliness, relaxation, motivation, and socialization in older people who have interacted with companion robotic animals [12,13,15-17].

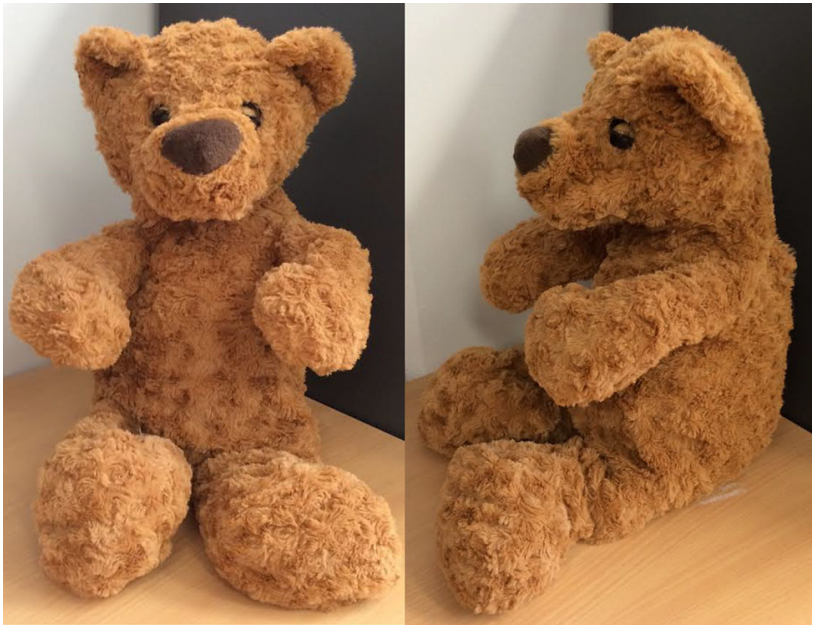

Fig. 1 CuDDler

Robotic animals may also improve mood. However, to date there has been limited research exploring the effect of robotic animals on mood.

The research reported in this paper concerns a new companion robot called CuDDler. The CuDDler robot is a prototype robotic teddy bear developed by Dr Tan Yeow Kee, Scientist and Group Leader of Robotic Senses Research Institute, for Infocomm, Singapore. CuDDler is $40 \mathrm{~cm}$ tall, $20 \mathrm{~cm}$ wide, and weighs approximately $4 \mathrm{~kg}$ (see Fig. 1). CuDDler has three degrees of freedom to move its neck, two degrees of freedom to move its arms and one degree of freedom to move its eyelids. CuDDler moves its limbs and vocally interacts with a low level bear like growl. Three contact microphones are positioned in CuDDler's head, stomach, and back to detect touch from a participant. The contact microphones identify the type of participant touch, for example, whether a participant hits, pats, strokes or squeezes CuDDler.

Two Android phones are used in the operation of CuDDler, and both phones are connected via a wireless-modem. One of the phones is placed inside CuDDler's back and this phone executes a software module that analyses the pattern of each participant's touch as they interact and touch CuDDler. The software module controls CuDDler's motion, gestures, and voice, and enables CuDDler to respond appropriately to the pattern and type of touch. For instance, when CuDDler's head is patted CuDDler responds with a happy gesture [18]. $\mathrm{CuDDler}$ emits a soft purring sound, blinks its eyes, moves its head up and down, and invites a hug from the participant through animated arm movements.

The second Android phone is external to CuDDler. If desired this phone can be used to send instructions to the internal phone inside CuDDler to manually control $\mathrm{CuD}$ Dler's response, motion and voice. In this study the research facilitator did not use the external phone and software module to manually control CuDDler's response. 


\section{Research Method}

\subsection{Design}

This study aimed to generate pilot data to explore the feasibility and effect of using an engaging robotic animal, namely CuDDler, on emotional states of people with dementia living in nursing home care. Such a population is at risk of limited social interaction and therefore loneliness and social isolation. The Griffith University Human Research Ethics Committee gave approval for the study, and informed consent was sought from the person's guardian and assent was sought from each participant at the time of each session.

The pilot study used a case-study design [19] to allow each individual's experience with CuDDler to be documented over time. Case study methodology is advantageous as it allows for close inspection and exploration of the feasibility and preliminary effects of using CuDDler. The project brought together experts from nursing, psychology and engineering/IT to understand the effect of CuDDler.

Specific research questions related to the study aim were developed and included the following:

1. How feasible, effective, and tolerable is the CuDDler robot for people with dementia in a nursing home setting?

2. What emotional response does the person with dementia display during CuDDler sessions?

3. What effect does CuDDler have on agitation in people with dementia?

\subsection{Setting}

The management from one nursing home setting in the west of Brisbane, Australia, agreed to participate in the study. The facility offers 107 residential care beds.

\subsection{Participants}

A purposive sample of older adults with a diagnosis of dementia was recruited. The sample comprised of five female participants. Residents were eligible to participate if they had a diagnosis of dementia, and they were physically able to sit with and hold CuDDler.

\subsection{CuDDler Intervention}

The intervention design, length and type were based on the team's previous research with companion robots, which suggested that short, individual sessions are more likely to encourage engagement [12]. The process of giving and removing CuDDler to and from each participant was standardised and guided by a protocol that is outlined below.

\subsubsection{Aim}

The sessions introduced CuDDler and were used to help participants to see the potential of CuDDler. As CuDDler is a companion robot designed to interact and respond to touch, participants were asked to freely interact with CuDDler.

\subsubsection{Participants}

Each session included one participant, one facilitator and one CuDDler.

\subsubsection{Timing and Length}

Participants were offered three researcher-facilitated CuDDler sessions per week for $30 \mathrm{~min}$ each, for a period of 5 weeks (a total of 15 sessions or $7.5 \mathrm{~h}$ ). The sessions were conducted between 13:00 and 17:00 - the time when people with dementia are known to be most agitated $[17,20]$. This time was deliberately chosen in order to examine the effectiveness of CuDDler in reducing behavioural and psychological symptoms of dementia, such as agitation.

\subsubsection{Facilitator}

The facilitator of each intervention (MB) is a registered nurse with extensive experience in dementia care. As a research team member MB was aware of the research aim. The facilitator's role was to observe participants and to direct participants to interact with CuDDler using a series of questions that referred to CuDDler and the resident's experience with CuDDler.

\subsubsection{Setting}

All sessions were conducted in a quiet and closed room in the nursing home.

\subsubsection{Recording}

Two GoPro cameras were used to record each intervention session. One camera was positioned behind CuDDler to record participant's facial expression and the second was positioned beside CuDDler to capture the participant's body movement and interaction with CuDDler.

\subsubsection{Procedure}

The facilitator ensured each participant was directly in front of $\mathrm{CuDDler}$ and asked them to touch and speak with CuDDler so that they could see CuDDler's response. 
1. Introduction Each session involved an introduction to CuDDler and a discovery element including a review of CuDDler's emotions, social interaction, and closure. During the introduction CuDDler was introduced to each participant with a statement of "Hello participant. This is CuDDler. CuDDLer is a robotic bear. Would you like to get to know CuDDler?"

2. Discovery This was followed by the opportunity for discovery, whereby the participant was encouraged to touch and interact with CuDDler and to explore CuDDler's emotions. Where a participant seemed unsure or unable to touch or speak to CuDDler the facilitator assisted CuDDler's reaction by helping the participant to touch CuDDler. Participants were encouraged to follow CuDDler's movements and to watch CuDDler's eyes when they spoke to CuDDler.

3. Engagement The facilitator then further engaged each participant with CuDDler through the use of the following questions: What does CuDDler remind you of? What would you like to do with CuDDler? Would you like the facility to have a CuDDler? Do you like the feel of $\mathrm{CuD}$ Dler's fur? What is good about CuDDler? What is not so good about CuDDler?

4. Closure At the end of the session the facilitator thanked the participant for their participation and informed them that CuDDler would be back later in the week.

\subsection{Outcome Measures}

At baseline, the mini-mental state examination (MMSE) [21] was administered to detect each participant's level of cognitive function. The MMSE's maximum total score is 30 . College education is known to influence screening assessment of cognitive impairment. Therefore, in the early stages of dementia the MMSE may not be sensitive enough to pick up cognitive impairment where a person has had a college level education [22]. This can explain why participants can have a high score on the MMSE but also have a diagnosis of dementia. Staff responsible for the care of each of the five participants were trained by the research team (MB) to complete the Cohen-Mansfield agitation inventory (CMAI, long-form) [22], for each resident twice; once prior to the $\mathrm{CuDD}$ ler intervention and also once post the CuDDler inter- vention. A maximum of two staff completed the CMAIs. The CMAI assesses the frequency with which residents manifest up to 29 behaviours, as observed by care staff over the past 2 weeks, on a 7-point rating scale. Higher scores indicate a higher level of frequency of agitated behaviours.

Semi-structured interviews were conducted with participants at the completion of the study. The intervention facilitator conducted the interviews, as she was familiar to participants. The team perceived that participants would respond to a familiar person more readily than they would to a stranger. Participants were asked a series of questions focused on their perceptions of CuDDler (see Table 1).

\subsection{Data Analysis}

Descriptive statistics were used to report demographic data, including severity of cognitive impairment. Thematic analysis was used to code the qualitative interview data according to the interview questions with an emphasis placed, where possible, on issues relating to feasibility, effectiveness, tolerability and reliability. Noldus ObserverXT 11.5 (www.noldus.com) was used to analyse the video data recordings.

\subsubsection{Video Coding Protocol}

A video coding protocol developed by members of the team was used to analyse participants' facial emotional responses, verbal engagement, visual engagement, behavioural engagement, and agitation during the intervention sessions. The coding protocol was based on the theory of emotional engagement [23] and behavioural engagement [24,25]. The coding protocol was used to code each individual participant's engagement with CuDDler at the time it occurred on the video.

The video coders (BS, CJ) viewed the recordings of the first intervention session for each participant without conducting any analysis. This initial viewing allowed the coders to calibrate their analysis for each individual resident, thus enhancing the reliability and validity of the analysis. The coders then individually coded each recording in a random order. Inter-rater reliability of the video analyses between the two coders was computed by comparing the

Table 1 Interview guide

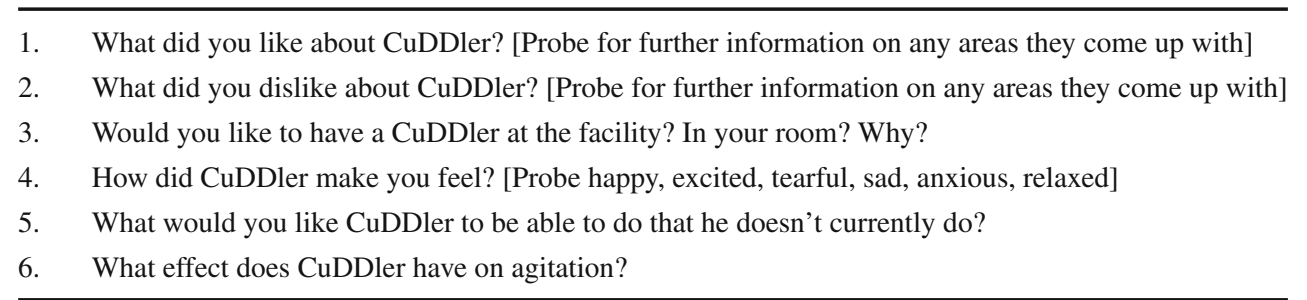


frequency and the sequence of behaviours coded within a one-second-tolerance interval. The inter-rater reliability was exceptionally high $(99.5 \%)$ and is explained by the use of video analysis software that allows videos to be coded in millisecond intervals, the coders' familiarity with the coding protocol, and the coders' expertise in video coding.

All behaviours and emotional responses were measured in duration where the behaviours or emotional responses were observed. Video data for a specific behaviour or emotional response were coded as missing if it was uninterpretable (e.g., when residents moved off camera). Missing video data for each intervention session was minimal $(M=14 \mathrm{~s})$.

\subsubsection{Emotional Response}

The video coding protocol for emotional response was based on the 'Observed Emotions Rating Scale' (OERS) [23]. The participant's emotional response (i.e., facial expressions) to $\mathrm{CuDDler}$ was coded as either neutral, pleasure, anger, anxiety/fear, or sadness, and the duration of facial expressions was measured. Pleasure was considered as a positive emotion, with anger, anxiety/fear, and sadness considered negative emotions.

\subsubsection{Engagement (Visual, Behavioural, and Verbal)}

The duration of participants' engagement with CuDDler was measured by visual alertness, verbal, and behavioural engagement. For people with dementia, alertness and attention are indictors of non-verbal engagement [24]. Visual engagement was therefore measured as the duration for which the resident appeared visually alert, maintained eye contact with CuDDler, or turned and moved their body towards CuDDler. Participants were coded as being visually disengaged if eye contact was not targeted to CuDDler.

Drawing from the work of Clair [25], verbal engagement was measured by the duration for which participants participated in, responded to and maintained a conversation about or towards CuDDler. When the target of the conversation was the facilitator, the participants were coded as being verbally engaged toward the facilitator. Participants were coded as being verbally disengaged when the conversation was directed to others (i.e., they were distracted) or when they were not participating, responding, or maintaining a conversation.

Behavioural engagement was measured as the duration for which participants touched, held, stroked, or handled CuDDler in an appropriate way. Due to participant "J's" visual agnosia (an impairment in recognition of visually presented objects), an attempt to touch or handle CuDDler was also considered as evidence of behavioural engagement. The absence of these behaviours was classified as being behaviourally disengaged.

\subsubsection{Agitation}

We also measured the duration for which the participants showed signs of agitation. Drawing from the CMAI [26] codes, signs of agitation included restlessness, repeated movement, picking or fiddling with clothes, repetitive rubbing of limbs or torso, shouting and appearing anxious.

\section{Results}

\subsection{Participants}

The five female participants all had a documented diagnosis of dementia and they ranged from mild ("S" and "E) to a moderate ("A", "G", "J") stage dementia (see Table 2). The participants exhibited frequent agitation with scores on the CMAI between 38 and 81 pre-intervention (higher score indicates more frequent agitation) and between 36 and 97 post-intervention. There was also wide variation in the total duration of the intervention across the sessions. This ranged from $19.70 \mathrm{~min}$ (“E”) to $88.75 \mathrm{~min}$ ("J") (see Table 2).

\subsection{Participant Case Studies}

The results are presented below as individual case studies. Participants' behavioural and emotional responses to CuDDler are summarised in Table 3. A case study of "J" is first presented as she exhibited the most positive response toward CuDDler despite her visual agnosia. The next case studies are those of participants with mild dementia ("S" and "E") and are followed by those with moderate stage dementia ("A" and "G").

\subsubsection{Participant "J"}

Staff and family were keen for "J" to be involved in this study as her highly anxious and distressed state was a daily challenge for staff and they thought CuDDler might comfort "J".

Engagement Despite her daily agitated state and visual agnosia, "J" exhibited a high level of visual, verbal, and behavioural engagement across the intervention sessions. " $\mathrm{J}$ " attended 10 out of the 15 sessions and, of all the participants, she spent the longest time interacting with $\mathrm{CuDDler}$. On average, she engaged with CuDDler visually for 480 s, verbally for $438 \mathrm{~s}$, and behaviourally for $333 \mathrm{~s}$ during each session. By working and talking with the facilitator, she actively explored $\mathrm{CuDDler}$, its shape, responses and functions, and she tried to conceptually piece together the different parts of CuDDler to form a holistic impression of CuDDler's appearance. In Session 13, CuDDler experienced technical problems and was 


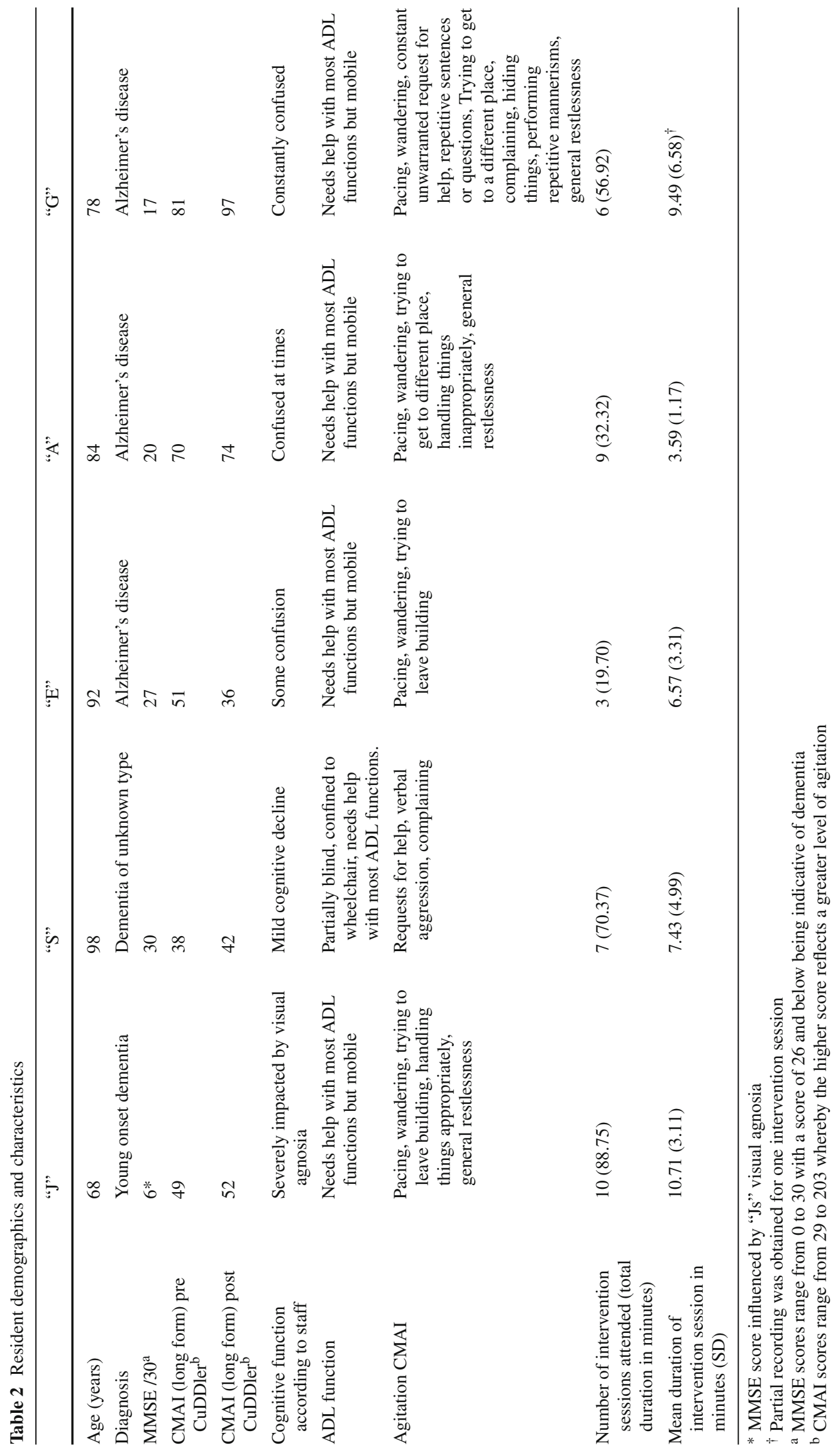


Table 3 Average duration in seconds of participants' behavioural and emotional responses to CuDDLer

\begin{tabular}{|c|c|c|c|c|c|c|c|c|c|}
\hline Participant & $\begin{array}{l}\text { Session } \\
\text { duration }\end{array}$ & Neutral & $\begin{array}{l}\text { Positive } \\
\text { emotion }\end{array}$ & $\begin{array}{l}\text { Negative } \\
\text { emotion }\end{array}$ & $\begin{array}{l}\text { Visual } \\
\text { engagement } \\
\text { with CuDDler }\end{array}$ & $\begin{array}{l}\text { Verbal } \\
\text { engagement } \\
\text { with CuDDler }\end{array}$ & $\begin{array}{l}\text { Verbal } \\
\text { engagement } \\
\text { with facilitator }\end{array}$ & $\begin{array}{l}\text { Behavioural } \\
\text { engagement }\end{array}$ & Agitation \\
\hline $\mathrm{J}$ & 643 & 558 & 49 & 36 & 480 & 438 & 26 & 333 & 0 \\
\hline $\mathrm{S}$ & 446 & 398 & 38 & 10 & 209 & 163 & 133 & 14 & 4.87 \\
\hline $\mathrm{E}$ & 394 & 352 & 32 & 11 & 230 & 270 & 73 & 152 & 33 \\
\hline A & 216 & 184 & 26 & 6 & 82 & 66 & 86 & 4 & 10 \\
\hline G & 569 & 469 & 86 & 15 & 184 & 220 & 306 & 100 & 75 \\
\hline Average & 453 & 392 & 46 & 16 & 237 & 231 & 125 & 120 & 25 \\
\hline
\end{tabular}

unable to move. Despite her visual agnosia, "J" attempted to help the facilitator to fix and "wake up" CuDDler by talking to and pressing different parts of CuDDler.

Agitation and Emotional Response "J" exhibited few signs of agitation while she was engaging with CuDDler. Her facial expressions (smiles and laughter) suggested she found the interaction with CuDDler positive. She exhibited minimal negative emotions during the sessions despite the fact that when she was not with CuDDler she constantly cried, expressed her sadness, and wandered aimlessly. In many instances, "J" displayed pleasure when CuDDler responded to her touch and when she talked about CuDDler and her dog that was recently deceased. CuDDler gave "J" the opportunity to reminiscence about her dog in a quiet one-on-one session. She often talked about others in the facility not understanding her. Her anxiety, which was a major concern for those around "J", was reduced during the sessions and this seemed to result in "J"s communication being clearer during each session. Although as a result of a visual perceptual deficit" J" had difficulty in detecting the contours of CuDDler and interpreting what she could see, she did not exhibit any fear or anxiety towards it. There was a slight increase in the frequency of agitation displayed by "J" according to the CMAI from pre-intervention (49) to post-intervention (52) and this may have been related to "J" looking for more interaction from the sessions.

\subsubsection{Participant " $S$ "}

Engagement "S" was very willing to try CuDDler. Although she only attended 7 of the 15 sessions she spent the second longest duration with CuDDler (70.37 min, see Table 2). During the first week of the trial, "S" exhibited a moderate level of engagement with CuDDler. However, her engagement gradually declined over the course of the intervention. On average, visual, verbal and behavioural engagement with the CuDDler was observed for 209, 163 and 14 s during each session, respectively. "S"s engagement was largely verbal in nature. She frequently asked and talked about the purpose of using CuDDler. Towards the end of the intervention (Session 10 and 11), "S" exhibited minimal to no interaction with $\mathrm{CuDDler}$ and directed her verbal engagement to the facilitator.

Agitation and Emotional Response "S"s facial expressions were consistent with her mood on the days of the intervention. In some sessions, she exhibited a neutral expression with no positive or negative emotion. In other sessions, she exhibited a high level of pleasure. She stated that CuDDler was a "toy for children" and that she was "too old for it". Despite "S"s attitude towards CuDDler, there was a degree of novelty for "S" but CuDDler did not offer enough responses or functions to keep "S"s interest. There was a slight increase in frequency of agitation, according to the CMAI, from pre-intervention (38) to post-intervention (42).

\subsubsection{Participant " $E$ "}

Engagement Out of the five participants, "E" attended the least number of intervention sessions $(n=3)$ due to poor health. However, she showed relatively high visual (230s), verbal (270s) and behavioural (152 s) engagement with CuDDler during the sessions she did attend. "E" was keen to interact with CuDDler by frequently stroking and talking to it.

Agitation and Emotional Response On average "E" exhibited evidence of agitation for about $33 \mathrm{~s}$, which was higher than the other four participants. She was highly distracted by unexpected noises outside her room and the duration of her agitation also increased as she progressed through the three sessions. However, "E" exhibited pleasure when she was talking to $\mathrm{CuDDler}$ and when $\mathrm{CuDDler}$ responded to her. She also displayed minimal negative emotion during the session. "E" also demonstrated a reduction in agitated behaviours, according to CMAI, from 51 pre-intervention to 36 post-intervention. However, this drop may have been related to her ill health, which had resulted in a reduced level of activity. 


\subsubsection{Participant " $A$ "}

Engagement "A" attended 9 out of 15 sessions and indicated that she was not fond of CuDDler. On average, she visually and verbally engaged with CuDDler for 82 and $66 \mathrm{~s}$, respectively, during each session. She also spent $4 \mathrm{~s}$ behaviourally engaging with $\mathrm{CuDDler}$. Furthermore, her engagement with CuDDler declined as she progressed through the sessions. In the last two sessions, "A" largely ignored CuDDler and instead directed her communication toward the facilitator.

Agitation and Emotional Response Despite her low level of engagement with CuDDler, "A" expressed minimal negative emotions and agitation during the sessions. She exhibited relatively more pleasure and less negative emotions during the first two sessions. Occasionally, she would laugh and talk to $\mathrm{CuDDler}$ in response to CuDDler's growl and movements. However, she refused to hold CuDDler on her lap and said that she was "too old" to interact with CuDDler. At the start of the trial, she regularly commented that CuDDler was "beautiful" but that "I have a son and he will love this (CuDDler), but not me". There was a slight increase in the frequency of agitation according to "A's" CMAI from pre-intervention (70) to post-intervention (74).

\subsubsection{Participant " $G$ "}

Engagement "G" attended 6 out of 15 sessions and appeared to enjoy the facilitator's company more than CuDDler. She was very keen to speak to and engage with the facilitator. She would regularly interact with CuDDler for several seconds and then continue her conversation with the facilitator. On average, she showed moderate levels of visual (184s) verbal (220s) and behavioural (100 s) engagement with CuDDler during sessions. Her behavioural engagement largely consisted of holding and moving CuDDler's feet and hand, which were the softer parts of CuDDler. "G" commented that she liked CuDDler's feet because "they are soft and cuddly". In Session 6, CuDDler experienced technical difficulties and was not moving. "G"s visual, behavioural, and verbal engagement with CuDDler was noticeably lower in that session.

Agitation and Emotional Response " $\mathrm{G}$ " exhibited a high level of agitation and was easily distracted in the sessions. "G" had the highest CMAI score pre-intervention (81) and this score increased during the study (97). She also experienced physical illness during the study and this may have influenced her response to CuDDler. In most of the sessions, she rocked on her chair back and forward, fiddled with her clothing, repeatedly scratched her face, and constantly looked around the environment. She was also disorientated; in one session, she told the facilitator that she did not like working in the facility. Her agitation ceased whenever she initiated engagement with CuDDler or when the facilitator directed her attention to $\mathrm{CuDDler}$ by asking her questions about $\mathrm{CuD}$ Dler. "G"s facial expression was mostly positive during the sessions. On average, she displayed pleasure for $86 \mathrm{~s}$ during a session, the highest amount of all the participants. She would always smile and laugh when she saw CuDDler move and when she talked to CuDDler. She would also laugh when she was talking to the facilitator. Most of the negative emotions for " $G$ " were observed when she complained about her experience in the residential facility. " $G$ " always praised CuDDler as "beautiful", "gorgeous", "sweet" and "lovely bear". She regularly called CuDDler a "sweetheart" and "darling".

\subsection{Feasibility}

There were several technical challenges encountered during the use of CuDDler. The preparation and setup of $\mathrm{CuD}$ Dler for each testing session was time-consuming due to the need to set up the two Android phones, connect the phones onto the wireless modem and run the software module before attaching one of the phones inside CuDDler. These procedures typically required at least $10-15 \mathrm{~min}$. The attachment of the phone inside CuDDler's back also made troubleshooting any problems with the software module or the connection between the phones very challenging. The connection between the two phones was unstable at times and disconnection was regularly experienced. When connection issues occurred, CuDDler also made a very loud and disturbing motor sound while continuously twisting his arm. This agitated participants during some of the testing sessions. Furthermore, the motors and mechanical joints on $\mathrm{CuD}$ Dler's neck and limbs were fragile and resulted in CuDDler's neck and left limb being broken during interactions with one participant. Lastly, CuDDler's speaker could not be turned off between sessions. This quickly drained the battery and resulted in several sessions where CuDDler made no sound.

These technical difficulties were a challenge for the facilitator in trying to maintain the same intervention type and duration for each participant. However, the technical problems were readily solved, and were of short duration (an average of $10 \mathrm{~min}$ ). They did, however, in two separate sessions stop CuDDler from interacting with participants. The video observations indicate the participants did not appear to be affected by these technical problems and they either continued interacting with CuDDler, even though $\mathrm{CuDDler}$ was not moving, or waited for the researcher to resolve the problems.

\subsection{Qualitative Interviews}

Interviews were conducted with the study participants one week after the CuDDler intervention was completed in December 2013. Individual interviews with the facilitator 
were from 10 to $15 \mathrm{~min}$ duration and were digitally recorded and then transcribed verbatim. When asked, all participants said they remembered their interactions with CuDDler. Although it was challenging to keep participants focused on the interview questions, their answers to the questions were consistent with the comments made during the intervention sessions. The results are presented according to the topics of feasibility, tolerability, and effectiveness.

\subsubsection{Feasibility}

When answering the first question: "What did you like about CuDDler?" "A" thought it was "nice to see it. The way it moves its arms and legs" but she did not show much interest in CuDDler overall and did not respond to further probing. "J" said she liked it because it was "something to cuddle, the different positions, the way he moves." Consistent with her visual agnosia "J" attempted to explain the movement of CuDDler in association with her pet dog, which was important for her in her life. "G" thought CuDDler just needed some "tweaking" and there was nothing she really disliked. However, during the intervention session, "G" commented that CuDDler needed to be more "cuddly and soft like its feet". "E" described CuDDler as "too mechanical" and "looks a bit dead". She also found it to be "very heavy and it is difficult to hold him up". "S" also had similar concerns and said CuDDler was too heavy to hold and preferred CuDDler to sit on the table rather than in her lap.

\subsubsection{Tolerability}

There were varying comments in relation to the participants' perceptions and their tolerability of CuDDler. These comments were made in answer to the question: "Would you like to have a CuDDler at the facility?" Throughout the interview "S" was consistent in her reply that she only liked the bear as she thought it was "great for children." "S" explained that she "was too old for toys like that. I don't think it has a lot of use for adults. I don't think it appeals to adults. They might think it's too much of a toy for adults" "G" thought the bear was "lovely, he's a cute little bear." However, she also stated: "I would be terrified to pick one up if I had one somewhere else." She then proceeded to talk about her son in New York and it was difficult to refocus her on the question. "E" who, despite having a collection of teddy bears, expressed her lack of interest in CuDDler as, "the name CuDDler is a bit common. He's a bear and I have a few bears. I don't really want another bear. I haven't bought any bears; they have all been given to me. Bears are for boys". "E" had also expressed this sentiment during the intervention. In answer to the question: "Would you like to have a CuDDler in your room?" "A" explained that she would not like the bear in her room. She said she would be happy to have her son in her room but not the bear, because it "is just a doll, nothing special to me." "J" on the other hand could "imagine having him in my room", whilst "G" said: "No I couldn't, I'd be scared stiff". Consistent with her level of disinterest "S" replied "Not really". "E" also made an interesting comment that: "He's not a toy as such. Is he making those movements himself? I don't understand what they have put in him to make him work". "E" also assumed that the researchers were trying to sell CuDDler to her and therefore often refused to come to sessions.

\subsubsection{Effectiveness}

In answer to the question: "How did CuDDler make you feel (happy, excited, tearful, sad, anxious, relaxed)"? "A" thought it made her feel " Alright, why not? [It] an be calmer". But she also said that it could not do more because "It's just a piece of rug". "J" explained that: "It made me cry when it was sitting on my lap. I don't remember how it feels. It makes me feel relaxed but I cannot see it very well. He is cute, nice". "S" thought it did not make her feel any different and it was "a bit of a waste of money". "E" said, "Doesn't make me feel any different". "G" and "E" gave no indications of the effect of CuDDler on their emotional state. All of the participants lost interest in further questions about CuDDler at this stage and therefore the interviews were terminated.

\section{Conclusions and Discussion}

The aim of the study was to investigate the effect of $\mathrm{CuD}$ Dler on engagement and emotional responses of people with dementia living in LTC. CuDDler was developed in Singapore and designed for older people. The idea of a companion robot to support and engage older people and to reduce loneliness is one that remains controversial. In this case it appears that the robot development would have benefited from end user input into the design and functionality. Despite their cognitive decline participants raised a number of concerns regarding the feasibility and tolerability of CuDDler. According to the participants, the effectiveness of CuDDler for improving their engagement was also limited.

Feasibility and tolerability are crucial to the success and effectiveness of a robot $[27,28]$ and in this project not all participants accepted CuDDler. The developer chose the form of a teddy bear because he believed older people would have fond memories of being with a teddy bear. The developer may have also been influenced by the stereotype of older people with dementia being 'child-like' and therefore more accepting of a toy like animal. This study also demonstrates the importance of companion robots being an appropriate size and shape for older people. CuDDler was too rigid, too big and too heavy for these older people to place on their lap 
or to hug and, as a result, the potential for them to connect with $\mathrm{CuDDler}$ was reduced.

The acceptability of a robot as a companion demands that the users identify some of the attributes of a true living companion in the robot. The attractiveness depends on the robot being considered by the end users to have the properties of a small bear like character; able to make movement on its own, with potential for interaction, and where use and engagement is different and worth investing time. A successful robot should be regarded as worth exploring, and having the ability to interact with humans. There is some evidence of this discussed by the participants such as in the comment "it's lovely, he's a cute little bear". However, to a larger extent the participants identified CuDDler as a machine, a "piece of rug", "a toy", "too mechanical", and "a doll". Our results here open further avenues to explore the extent that older people with dementia perceive the need for companionship from a robot and detach from the notion of the companion animal being a machine.

The video analysis of the sessions indicated that responses varied between participants and over time for each individual. Some of the participants' responses demonstrated that they were frustrated with CuDDler and the way it performed (or failed to perform). To ensure a successful interaction between humans and robots, it has been argued that the robot must behave in a way that can be intuitively understood by humans [29]. Furthermore, its appearance must match the functions it performs [30]. Although a teddy bear might evoke fond memories of childhood these participants identified CuDDler as being too toy like-it looked like a teddy bear they might have had as a child — and therefore it was not something they deemed as being appropriate for older people to identify with. As a teddy bear CuDDler may have evoked feelings of stigmatisation and infantilisation for four of the participants.

Participant " $J$ " who has visual agnosia had more engagement with CuDDler than the other participants. CuDDler became a companion for "J" or at least displayed the attributes of a companion. Participant "J" could not visualise $\mathrm{CuDDler}$ and therefore engaged with the touch and soft feel of CuDDler, and in particular his soft paws and legs. Rather than evoke thoughts of CuDDler being a toy, the texture and sensation of the fur reminded "J" of her recently deceased dog. In this case, CuDDler evoked fond memories of the animal she missed holding and touching. The one-onone session also gave her the opportunity to talk about her feelings. We speculate that if older people are not cognitively impaired, the less companionship attributes are assigned and portrayed to zoomorphic robots [31].

There is an increasing recognition of the need for older people with dementia to engage in meaningful relationships and have opportunities for dialogue [5]. While CuDDler had differing effects on the five participants it was also clear that $\mathrm{CuDDler}$ provided the opportunity for the participants to engage in social interaction. Engaging people with dementia in play is important in improving mood and quality of life [32]. CuDDler provided the opportunity for participants to be playful as well as to laugh with and at CuDDler. Even when CuDDler had technical problems such as being slow to react or the leg falling off, participants continued to speak to $\mathrm{CuDDler}$ and/or the facilitator. In this way, CuDDler provided a platform for social interaction. Over time, however, the facilitator became the source of engagement for some participants rather than CuDDler and this raises the question as to whether the human element may have been all that was required to engage the participants.

There is a vast body of literature on the ethical implications of using social robots for older people [33-35]. Although a number of these concerns relate to offering robots for companionship, in this study one of the key concerns for the researchers was taking CuDDler away from " $\mathrm{J}$ " at the end of the study, as CuDDler appeared to have a therapeutic effect on "J". "J"s family and staff were prepared for this situation and a replacement source of conversation for "J" was sought at the end of the trial.

The small number of participants limited this study, but the methodological approach, standardised intervention and systematic analysis were its strengths. The facilitated sessions were necessary in this situation as CuDDler was a prototype and we were unsure how participants would react to or manage CuDDler, particularly if the technology broke down. However, the researchers' recent research [12] and a current study [36] suggest that a non-facilitated session would allow for a clearer indication of the effect of companion robots when a non-prototype robot is being tested.

The study was also limited by technological problems in relation to the android phone not working. Furthermore, CuDDler's leg fell off and its head became unstable at one stage in the study. However, these problems did not occur in each session and so may have had little impact on participant's engagement. "J" for example became engaged in trying to "wake up" CuDDler when it was not working.

The varied amount of time spent with CuDDler limits our ability to make inferences about the dose-response effects of companion robots. This variability, however, was influenced by participant health and environmental factors, and so was beyond our control. We aimed to overcome the common problem of engaging residents with dementia in research by using one facilitator with a background in dementia, having the facilitator collect participants for each session, and the facilitator engaging participants in conversation about $\mathrm{CuD}$ Dler. We are mindful, however, that the close presence of the facilitator may have promoted comfort in the person with dementia [37] and thereby positively influenced participants' responses to CuDDler.

There is a clear need for further studies on the development and use of companion robots. In particular there is the need 
to include older people, people with dementia and carers in the development of companion robots to ensure that the design is appropriate and feasible for end users. In addition, the development of companion robots must take into account the heterogeneity of older people with dementia. This study clearly showed that the five participants had varying opinions of CuDDler.

In keeping with our premise that robots are poor substitutes for human contact, there is also a need to directly compare the benefits of companion robots and human interaction. Participant "J", for example, felt anxious and misunderstood when surrounded by people, but exhibited a very favourable response to CuDDler. Being able to talk to and about $\mathrm{CuD}$ Dler while remembering her deceased dog gave "J" comfort and reduced her anxiety, as she focused on verbalising her visual interpretation of the robot. Although CuDDler was far from perfect as a companion robot, CuDDler brought joy and pleasure to "J" that no human activity (staff or family) in the nursing home had been able to achieve. Finally, pilot studies such as the current study are needed to inform the development of robots, and if successful, the pilot data can be used to inform larger high quality studies, research effort, and industrial development.

Acknowledgments The authors thank Pam Scherman Carr, staff and residents of RSL Care Pinjarra Hills, Brisbane, Australia for their participation in this project, Dr Tan for giving us CuDDler to trial and Dr Rene Hexel and Carl Lusty from IIIS at Griffith University for undertaking maintenance of CuDDler. The Dementia Collaborative Research Centre - Carers and Consumers, Australia, funded this study.

\section{Compliance with ethical standards}

Conflict of Interest No conflict of interest declared.

Ethical Statement The study received ethical approval from Griffith University Human Research Ethics Committee.

\section{References}

1. World Health Organisation (WHO), Alzheimer's Disease International (2012) Dementia. A public health priority. World Health Organisation, Geneva

2. Australian Institute of Health and Welfare (2012) Dementia in Australia. Australian Institute of Health and Welfare, Canberra

3. Draper B (2011) Understanding Alzheimer's \& other dementias. Longueville Books, Woollahra

4. Brodaty H, Draper BM, Low LF (2003) Behavioural and psychological symptoms of dementia: a seven-tiered model of service delivery. Med J Aust 178:231-234

5. Moyle W, Venturato L, Griffiths S, Grimbeek P, McAllister M, Oxlade D, Murfield J (2011) Factors influencing quality of life for people with dementia: a qualitative perspective. Aging Ment Health 15:970-977

6. Hwang SS, Kim Y, da Yun Y, Kim YS, Jung HYJ (2012) Exploration of the associations between neurocognitive function and neuroleptics side effects. Psychiatr Res 46:913-919

7. Vickland V, Werner J, Morris T, McDonnell D, Draper B, Low LF, Brodaty H (2011) Who pays and who benefits. How different mod- els of shared responsibilities between formal and informal carers influence projections of costs of dementia management. BMC Pub Health 11:793

8. Vernooiji-Dassen M, Vasse E, Zuidema S, Cohen-Mansfield J, Moyle W (2010) Psychosocial interventions for dementia patients in long-term care. Int Psychogeriatr 22(7):1121-1128

9. Harmer BJ, Orrell M (2008) What is meaningful activity for people with dementia living in care homes? A comparison of the views of older people with dementia, staff and family carers. Aging Ment Health 12:548-558

10. Brooker D (2008) What makes a life worth living? Aging Ment Hlth 12:525-527

11. Libin A, Cohen-Mansfield J (2004) Therapeutic robocat for nursing home residents with dementia: preliminary inquiry. Am J Alzheimer's Dis Other Demen 19:111-116

12. Moyle W, Cooke M, Beattie E, Jones C, Klein B, Cook G, Gray C (2013) Exploring the effect of companion robots on emotional expression in older people with dementia: a pilot RCT. J Gerontol Nurs 39:46-53. doi:10.3928/00989134-20130313-03

13. Wada K, Shibata T (2007) Living with seal robots: its sociopsychological and physiological influences on the elderly at a care house. IEEE Trans Robot 23:972-980

14. Fluffy baby robot helps keep you company. http://www. newscientist.com/blogs/onepercent/2011/12/baby-robot.html. Accessed 11 April 2015

15. Frennert S, Ostlund B (2014) Review: seven matters of concern of social robots and older people. Int J Soc Robot 6:299-310

16. Sellers DM (2006) The evaluation of an animal-assisted therapy intervention for elders with dementia in long-term care. Act Adapt Aging 30:61-77

17. Kanamori M, Suzuki M, Tanaka M (2002) Maintenance and improvement of quality of life among elderly patients using a pettype robot. Nippon Ronen Igakkai Zasshi 39:214-218

18. Limbu DK, Anthony WCY, Adrian THJ, Tran AD, Kee CT, Tran HD, Alvin WHY, Terence NWZ, Jiang R, Li J (2013) Affective social interaction with CuDDler robot. In: 6th IEEE conference on robotics, automation and mechatronics (RAM), pp 179-184

19. Yin RK (2008) Case study research. Design and methods, 4th edn. Sage Publications, Los Angeles

20. de Jonghe SE, Korevaar AJC, Van Munster BC, de Rooij SE (2010) Effectiveness of melatonin treatment on circadian rhythm disturbances in dementia. Are there implications for delirium? A systematic review. Int J Geriatr Psychiatry 25:1201-1208

21. Folstein MF, Folstein SE, McHugh PR (1975) Mini-mental state: a practical method for grading the cognitive state of patients for the clinician. J Psychiatr Res 12:189-198

22. O'Bryant SE, Humphreys JD, Smith GE, Lynik RJ, Graff-Radford NR, Petersen RC, Lucas JA (2008) Detecting dementia with the mini-mental state examination in highly educated individuals. Arch Neurol 65:963-967

23. Lawton MP, Van Haitsma K, Klapper J (1996) Observed affect in nursing home residents with Alzheimer's disease. J Gerontol Ser B 51B:3-14

24. Cohen-Mansfield J, Dakheel-Ali M, Marx MS (2009) Engagement in persons with dementia: the concept and its measurement. Am J Geriatr Psychiatry 17:299-307

25. Clair AA (2002) The effects of music therapy on engagement in family caregiver and care receiver couples with dementia. Am J Alzheimer's Dis Demen 17:286-290

26. Cohen-Mansfield J, Werner P (1998) Predictors of aggressive behaviour: a longitudinal study in senior day-care centres. J Gerontol 53B:300-310

27. Heerink M, Kröse B, Evers V, Wielinga B (2010) Assessing acceptance of assistive social agent technology by older adults: the almere model. Int J Soc Robot 2:361-375 
28. Salvini P, Laschi C, Dario P (2010) Design for acceptability: improving robots' coexistence in human society. Int J Soc Robot $2: 451-460$

29. Kanda T, Miyashita T, Osada T, Haikawa Y, Ishiguro H (2008) Analysis of humanoid appearances in human-robot interaction. IEEE Trans Robot 24:725-735

30. Goetz J, Kiesler S, Powers A (2003) Matching robot appearance and behavior to tasks to improve human-robot cooperation. In: Proceedings of the 2003 IEEE international workshop on robot and human interaction communication. Millbrae, CA, pp 55-60

31. Bartlett B, Estivill-Castro V, Seymon S (2004) Dogs or robots: why do children see them as robotic pets rather than canine machines? In: Proceedings of the fifth conference on Australasian user interface, vol 28. Australian User-Interface Conference AUIC '04, Dunedin, New Zealand, Australian Computer Society, Inc., pp 714

32. Dunn J, Balfour M, Moyle W, Cooke M, Martin K, Crystal C, Yen A (2013) Playfully engaging people living with dementia: searching for Yum Cha moments. Int J Play 2:174-186. doi:10.1080/ 21594937.2013.852052

33. Lin P, Abney K, Bekey GA (2011) Robot ethics: the ethical and social implications of robotics. MIT Press, Cambridge

34. Sharkey A, Sharkey N (2012) Granny and the robots: ethical issues in robot care for the elderly. Ethics Inf Technol 14:27-40

35. Sharkey A, Sharkey N (2011) Children, the elderly, and interactive robots. IEEE Robot Autom Mag 18:32-38

36. Moyle, W, Beattie E, Draper B, Shum D, Thalib L (2014) Effect of an interactive therapeutic robotic animal on engagement, mood states, agitation and antipsychotic drug use. NHMRC Project Grant APP1065320, 2014-2017

37. Moyle W, Cooke ML, Beattie E, Shum DHK, O'Dwyer S, Barrett S, Sung B (2014) Foot massage and physiological stress in people with dementia: a randomized controlled trial. J Altern Complementary Med 20:305-311

Wendy Moyle is Professor and Director of the Centre for Health Practice Innovation in the Menzies Health Institute Queensland, Griffith University. She is also a research leader in the Dementia Collaborative Research Centre - Carers and Consumers. Her research focus is on quality of life of people with dementia and carers, and evidence based research to manage behavioural and psychological symptoms of dementia. She leads a social robotics laboratory at Griffith University where technologies including social robots are examined with end users such as older people, carers and health professionals.

Cindy Jones is a Research Fellow with Menzies Health Institute Queensland, Centre for Health Practice Innovation (HPI). She is a Griffith representative for the Queensland Dementia Training and Study Centre (QLD DTSC). The focus of her work includes an exploration of relationships in long-term care; expression of sexualities by older people; and quality of and evidence-based dementia care. This work is being developed around the key areas of knowledge transfer and workforce training and development. Dr Jones has also been an active member of a team, led by Professor Moyle, conducting cutting edge research on social robotics for end users such as older people, carers and professionals.
Billy Sung is a Research Assistant in the Centre for Health Practice Innovation in the Menzies Health Institute Queensland, Griffith University. Mr Sung is a member of a team, led by Professor Wendy Moyle, conducting innovative research that examines the use of social robotics and assistive technologies to improve the quality of life of older adults, especially people with dementia. His research also focuses on exploring different methods such as facial expression analysis to empirically and reliably measure people's behavioural and emotional responses toward different stimuli including social robotics.

Marguerite Bramble is a Project Manager in the Griffith University Centre for Health Practice Innovation, Menzies Health Institute Queensland. She is a Registered Nurse with extensive experience in education, research and clinical practice in dementia care. Her current role is focused on managing a large translational research project that examines the effects of an interactive robotic animal on behavioural and psychological symptoms of dementia and quality of life for people with dementia in residential aged care.

Siobhan O'Dwyer is a Research Fellow in the Centre for Health Practice Innovation, Menzies Health Institute, Griffith University. She has led world-first research on suicide and homicide risk in family carers of people with dementia. She is also a member of a research team, led by Professor Moyle, exploring the role of social robotics in dementia care and psychosocial approaches to managing the symptoms of dementia.

Michael Blumenstein is a Professor and Head of the School of Information and Communication Technology at Griffith University. He is a nationally and internationally recognised expert in the areas of automated Pattern Recognition and Artificial Intelligence. Professor Blumenstein's research also spans various projects applying Artificial Intelligence to the fields of Engineering, Environmental Science, Neurobiology and Coastal Management. He currently serves on the Australian Research Councilxs (ARC) College of Experts on the Engineering, Mathematics and Informatics (EMI) panel. In addition, he is a member of the Executive of the Australian Council of Deans of Information and Communication Technology (ACDICT). In 2009 he was named as one of Australiaxs Top 10 Emerging Leaders in Innovation in the Australianxs Top 100 Emerging Leaders Series supported by Microsoft. He is a Fellow of the Australian Computer Society and a Senior Member of the IEEE.

Vladimir Estivill-Castro is Professor of Information Technology and deputy-director of the Institute for Intelligent and Integrated Systems. He leads the Machine Intelligence and Pattern Analysis Laboratory (MiPal), securing Mi-Pal's participation at RoboCup's Standard Platform League (2002-2006 \& 2011-2014) and RoboCup@Home (2006-2007). He has a passion for software architecture for robotic systems. His recent work focuses on safe software for embedded and robotics systems, but also researched the use of robotic systems to assist people with disabilities as well as studying children's attitudes of people for robotic technology. He is Senior Member of the Association for Computing Machinery (ACM) and also senior member of the IEEE Computer Society. 